\title{
Gut microbiota-derived propionate reduces cancer cell proliferation in the liver
}

\section{LB Bindels', P Porporato², EM Dewulf', J Verrax ${ }^{3}$, AM Neyrinck', JC Martin ${ }^{4}$, KP Scott ${ }^{4}$, P Buc Calderon ${ }^{3}$, O Feron ${ }^{2}$, GG Muccioli ${ }^{5}$, P Sonveaux ${ }^{2,6}$, PD Cani ${ }^{1,6}$ and NM Delzenne*,1}

'Metabolism and Nutrition Research Group, Louvain Drug Research Institute, Université catholique de Louvain, Avenue Mounier 73, box B I.73.1 I 1200, Brussels, Belgium; ${ }^{2}$ Pole de Pharmacologie et thérapeutique, Institut de recherche expérimentale et Clinique (IREC), Université catholique de Louvain, Brussels, Belgium; ${ }^{3}$ Toxicology and Cancer Biology Research Group, Louvain Drug Research Institute, Université catholique de Louvain, Brussels, Belgium; ${ }^{4}$ Gut Health Division, Rowett Institute of Nutrition and Health, University of Aberdeen, Aberdeen, UK; ${ }^{5}$ Bioanalysis and Pharmacology of Bioactive Lipids lab, Louvain Drug Research Institute, Université catholique de Louvain, Brussels, Belgium

BACKGROUND: Metabolites released by the gut microbiota may influence host metabolism and immunity. We have tested the hypothesis that inulin-type fructans (ITF), by promoting microbial production of short-chain fatty acids (SCFA), influence cancer cell proliferation outside the gut.

METHODS: Mice transplanted with Bcr-Abl-transfected BaF3 cells, received ITF in their drinking water. Gut microbiota was analysed by I6S rDNA polymerase chain reaction (PCR)-denaturing gradient gel electrophoresis (DGGE) and qPCR. Serum Short-chain fatty acids were quantified by UHPLC-MS. Cell proliferation was evaluated in vivo, by molecular biology and histology, and in vitro.

RESULTS: Inulin-type fructans treatment reduces hepatic BaF3 cell infiltration, lessens inflammation and increases portal propionate concentration. In vitro, propionate reduces BaF3 cell growth through a cAMP level-dependent pathway. Furthermore, the activation of free fatty acid receptor 2 (FFA2), a Gi/Gq-protein-coupled receptor also known as GPR43 and that binds propionate, lessens the proliferation of BaF3 and other human cancer cell lines.

CONCLUSION: We show for the first time that the fermentation of nutrients such as ITF into propionate can counteract malignant cell proliferation in the liver tissue. Our results support the interest of FFA2 activation as a new strategy for cancer therapeutics. This study highlights the importance of research focusing on gut microbes-host interactions for managing systemic and severe diseases such as leukaemia.

British Journal of Cancer (2012) I 07, 1337-1344. doi:I0.1038/bjc.2012.409 www.bjcancer.com

Published online 13 September 2012

(c) 2012 Cancer Research UK

Keywords: gut microbiota; propionate; cancer cells; FFA2/FFAR2/GPR43; inulin-type fructans

The gut microbiota profits from its high metabolic potential for generating its own energy, mainly by fermenting dietary nondigestible carbohydrates (Neish, 2009). The carbohydrate fermentation end products are gases and organic acids, including lactate and short-chain fatty acids (SCFA) such as acetate, propionate and butyrate (Neish, 2009). In humans, butyrate constitutes a major energy source for colonocytes (Guarner and Malagelada, 2003). Propionate is mainly taken up by the liver, whereas acetate reaches peripheral tissues (Guarner and Malagelada, 2003). Short-chain fatty acids are considered key metabolic and immune cell regulators (Neish, 2009). Acetate and propionate exhibit antiinflammatory properties in human monocytes and in vivo in colitis models (Cox et al, 2009; Maslowski et al, 2009). In human cancer cells, butyrate and other SCFA affect the cell cycle by inhibiting proliferation and inducing differentiation and cell death (Siavoshian et al, 2000; Aoyama et al, 2010; Tang et al, 2011a).

\footnotetext{
*Correspondence: Professor NM Delzenne;

E-mail: nathalie.delzenne@uclouvain.be

${ }^{6}$ These authors contributed equally to this work.

Received 2 April 2012; revised 25 June 2012; accepted 15 August 2012; published online 13 September 2012
}

Intracellular mechanisms involved in cell proliferation and cell death (activation of caspases 3 and 7, and decreased histone deacetylase activity), have been extensively evaluated using in vitro assays (Aoyama et al, 2010; Tang et al, 2011a). More recently, two G-protein-coupled receptors, free fatty acid receptor 3 (FFA3) and FFA2, also known as GPR41 and GPR43, respectively, have been identified as receptors for SCFA (Brown et al, 2003; Le Poul et al, 2003). Propionate is considered the most potent endogenous agonist for both FFA3 and FFA2 (Brown et al, 2003; Le Poul et al, 2003). Free fatty acid receptor 2 expression occurs mainly in immune cells, but also in adipocytes, enterocytes and endocrine cells (Brown et al, 2003; Le Poul et al, 2003). Free fatty acid receptor 3 exhibits a widespread expression pattern (spleen, lymph nodes, bone marrow, adipose tissue and colon) (Brown et al, 2003; Le Poul et al, 2003). Lessons from FFA2 knock-out mice and smallinterfering RNA technology, revealed properties of propionate and acetate attributed to their FFA2-binding activity, for example, the resolution of the inflammatory response in a mouse model of colitis (Maslowski et al, 2009), neutrophil chemotaxis (Vinolo et al, 2011), glucagon-like peptide-1 secretion (Tolhurst et al, 2012) and pre-adipocyte differentiation (Hong et al, 2005).

Based on the theory that butyrate decreases proliferation and increases apoptosis of colon cancer cell lines (Siavoshian et al, 
2000; Tang et al, 2011a), a nutritional approach, that is, administering fermentable non-digestible carbohydrates, has been proposed as an adjuvant in the treatment of colon cancer (PoolZobel and Sauer, 2007). Interestingly, fermentable non-digestible carbohydrates might also be relevant to controlling tumour growth outside the gastrointestinal tract. We, and others, have described an oral administration of inulin-type fructans (ITF), which are non-digestible carbohydrates, that decreases the tumour size in liver or mammary cancer mouse models (Taper et al, 1997; Kondegowda et al, 2011). Inulin-type fructans are fermented by saccharolytic bacteria, leading to an increased production of SCFA in the mouse caecum (Busserolles et al, 2003). As a prebiotic nutrient, ITF change the composition and the activity of the gut microbiota (Everard et al, 2011), and control the host metabolism and immunity (primarily demonstrated in colitis, obesity and diabetes models) (Cherbut et al, 2003; Cani et al, 2006; Cani et al, 2007). Even so, no mechanistic study investigating ITF systemic anti-tumour effects has been performed so far.

We hypothesised that the increased SCFA production following ITF administration could hinder systemic cancer progression, and this could occur through FFA2 activation. We chose, as a model, murine proB $\mathrm{BaF} 3$ cells that ectopically express $\mathrm{Bcr}-\mathrm{Abl}$ because they can invade and proliferate in the lymphoid organs (Ren, 2005; Fiskus et al, 2006; Bindels et al, 2012), such as liver tissue, which actively takes up the SCFA that originates from the gut. Here, we demonstrate in vivo that ITF reduces $\mathrm{BaF} 3$ cell proliferation, increases propionate in the portal vein and lowers systemic inflammation, and in vitro that propionate reduces $\mathrm{BaF} 3$ cell proliferation through a cAMP level-dependent pathway and that FFA2 activation alters BaF3 cell growth.

\section{MATERIALS AND METHODS}

\section{Animals}

Female BALB/c mice (5-week-old, Charles River, France) were housed with two mice per cage in a 12-h light/dark cycle. Either a saline solution or BaF3 cells $\left(1 \times 10^{6}\right.$ cells in $0.1 \mathrm{ml}$ saline $)$ were injected into the tail vein of the anaesthetised mice. A day after the $\mathrm{BaF} 3$ inoculation, half of the mice that were transplanted with $\mathrm{BaF} 3$ cells received $0.2 \mathrm{~g}$ per day of ITF (Orafti p95, Beneo-Orafti, Oreye, Belgium) in the drinking water. The intake of food and water was recorded every 2 days to monitor the ITF consumption. The mice were killed at an advanced stage of the illness (13 days after the $\mathrm{BaF} 3$ inoculation) after anaesthesia with ketamine/xylazine i.p., 100 and $10 \mathrm{mg} / \mathrm{kg}$, respectively. Blood samples, liver, spleen and caecum content were harvested for further analysis. The experiment was approved by the local ethics committee, and the housing conditions were as specified by the Belgian Law of 6 April 2010, on the protection of laboratory animals (Agreement LA 1230314).

\section{Tissue and cells mRNA analyses}

Total RNA was isolated from the tissues and the cells using a TriPure Isolation Reagent Kit (Roche Diagnostics, Penzberg, Germany). Complementary DNA was prepared by reverse transcription of $1 \mu \mathrm{g}$ total RNA using a Reverse Transcription System Kit (Promega, Madison, WI, USA). Quantitative polymerase chain reactions (qPCR) were performed as previously described (Bindels et al, 2012). The primer sequences for the targeted genes are detailed in Supplementary Table 1.

\section{Gut microbiota analyses}

The gut microbiota composition was assessed by 16S rRNA gene analysis using denaturing gradient gel electrophoresis (DGGE) and qPCR, as previously described (Bindels et al, 2012). Genomic DNA was extracted from the caecal content using a QIAamp DNA
Stool Mini Kit (Qiagen, Hilden, Germany) according to the manufacturer's instructions. Full protocols are available online as Supplementary Methods.

\section{Biochemical and histological analyses}

The plasma L-lactate concentration was determined spectrophotometrically by measuring at $340 \mathrm{~nm}$ the increase of the $\mathrm{NADH}$-mediated absorbance in the presence of NAD + and L-lactate dehydrogenase (Roche, Mannheim, Germany) in a basic buffer. A spectrophotometric kit was used to determine the lactate dehydrogenase activity (Diasys, Sopachem, Brussels, Belgium). Plasma cytokines were measured using a customised multiplex kit (Bio-Rad, Nazareth, Belgium) and Luminex technology (Bio-Plex, Bio-Rad). The hepatic triglyceride content was determined using a spectrophotometric kit (Diasys) after lipid extraction with chloroform-methanol $(2: 1)$ according to Folch's method. To measure the hepatic glycogen content, liver tissue was digested in an alkaline medium and, after neutralisation, incubated in the absence or presence of amyloglucosidase. The glucose concentrations were determined for each condition with a spectrophotometric kit (Diasys), and the glycogen content was calculated as the difference in the glucose concentrations plotted to a standard curve. For the histological analysis, the formalin-conserved liver was embedded in paraffin.

\section{Short-chain fatty acids analysis}

Short-chain fatty acids were quantified using ultraperformance liquid chromatography coupled to mass spectrometry (UHPLC-MS) following extraction from serum and subsequent derivatisation. The extraction and derivatisation protocols were performed as described previously for valproic acid (Alric et al, 1981). Briefly, SCFA were extracted from $50 \mu \mathrm{l}$ of the portal serum with $\mathrm{HCl}$, acetonitrile and $\mathrm{KCl}$. After centrifugation, the upper phase was incubated with potassium carbonate. The derivatisation was performed with 2-bromo-2-acetonaphthone in the presence of 1,4,7,10,13-pentaoxacyclopentadecane and $\mathrm{K}_{2} \mathrm{CO}_{3}$. At the end of the reaction, the liquid phase was recovered and dried under a nitrogen stream. The resulting residue was dissolved in methanol. A 5- $\mu$ l aliquot was analysed by UHPLC-MS using an LTQ-Orbitrap Mass Spectrometer (ThermoFisher Scientific, Aalst, Belgium) coupled to an Accela UHPLC System (ThermoFisher Scientific). Analyte separation was achieved using a Hypersil Gold aQ $(50 \times 2.1)$ column (Thermo Scientific). The mobile phases $\mathrm{A}$ and B were composed of acetonitrile- $\mathrm{H}_{2} \mathrm{O}$-acetic acid 10:90:0.1 (v/v/ v) and acetonitrile-acetic acid 100:0.1 (v/v), respectively. The gradient $\left(0.5 \mathrm{ml} \mathrm{min}^{-1}\right)$ was designed as follows: 8 min of $90 \% \mathrm{~A}$; a transition from $90 \%$ A to $100 \%$ B linearly over $6 \mathrm{~min}$; followed by $3 \mathrm{~min}$ at $100 \% \mathrm{~B}$ and a subsequent re-equilibration at $90 \% \mathrm{~A}$. We performed MS analysis in the positive mode with an APCI ionisation source. Acetate, propionate and butyrate were quantified using valproate as an internal standard, and the calibration curves were prepared from pure standards diluted in water. The nature of the analytes was confirmed by their exact mass, MS2 fragmentation and co-elution with pure standards. Additionally, to reduce the acetate contamination, UltraPure Water (Cayman Chemical, Tallinn, Estonia) and HPLC-grade acetonitrile (VWR, Leuven, Belgium) were purified by SPE on StratoSpheres columns (PL-HCO3 MP-resin, Varian, Agilent Technologies, Santa Clara, CA, USA).

\section{Cell culture and chemicals}

The BaF3 cell line transfected with Bcr-Abl was a gift from $\mathrm{Dr}$ K Bhalla (MCG Cancer Centre, Medical College of Georgia, Augusta, GA, USA). The BaF3 cells were maintained in RPMI medium 1640 supplemented with 10\% foetal bovine serum (PAA 
clone, PAA, Pasching, Austria), streptomycin $100 \mu \mathrm{g} \mathrm{ml}^{-1}$, penicillin $100 \mathrm{IU} \mathrm{ml}^{-1}$ and $1 \%$ of non-essential amino acids solution (Gibco, Inchinnan, Scotland) at $37^{\circ} \mathrm{C}$ in humidified $5 \% \mathrm{CO}_{2}$. The generation of these cells is described in detail elsewhere (Fiskus et al, 2006). The human histiocytic lymphoma U937 (Sundstrom and Nilsson, 1976) and lymphoblast K562 cells were maintained in the same conditions, except for the non-essential amino acids solution.

All chemicals were purchased from Sigma-Aldrich, Saint Louis, MO, USA, except the synthetic FFA2 agonist (4-chloro- $\alpha$-(1-methyl ethyl)-N-2-thiazolyl-benzeneacetamide) (CMTB) (Wang et al, 2010) (Ambinter, Paris, France).

\section{Cell proliferation (MTT, manual counting, BrdU)}

The cell growth assay is based on metabolically active cells cleaving yellow thiazolyl blue tetrazolium bromide (MTT) to form purple formazan crystals. The formazan absorbance was measured at $570 \mathrm{~nm}$, from which a background value, measured at $650 \mathrm{~nm}$, was subtracted. Cell proliferation is expressed as a percentage of the value obtained for cells incubated with the vehicle (medium, PBS or DMSO, final concentration between 0.1 and $0.2 \%$ ). For manual counting, intact cells (determined by exclusion of erythrosine) were counted under a microscope in a Bürker cell. The bromodeoxyuridine incorporation assay was performed following the manufacturer's instructions (Cell Proliferation ELISA, BrdU colorimetric, Roche, Mannheim, Germany). All of the assays were performed in medium containing $10 \%$ foetal bovine serum. Complete protocols are available online as Supplementary Methods.

\section{Statistical analysis}

The results are expressed as the mean \pm s.e.m. Student's $t$-test, oneway ANOVA with Tukey post hoc test and two-way ANOVA with Bonferroni post hoc test were used for statistical analysis. $P<0.05$ was considered statistically significant (Graph-Pad Prism Software, San Diego, CA, USA).

\section{RESULTS}

\section{Inulin-type fructans administration decreases hepatic infiltration of BaF3 cells and systemic inflammation in mice}

$\mathrm{BaF} 3$ cells were transplanted into mice resulting in an aggressive malignancy that mimicked acute leukaemia and was characterised by an accumulation of $\mathrm{BaF} 3$ cells in the liver and spleen. The liver and spleen weights increased in the mice transplanted with $\mathrm{BaF} 3$ cells (BaF3 mice), in accordance with the fact that BaF3 cells infiltrate these organs (Figure 1A). Intriguingly, ITF administration impaired the liver weight gain induced by $\mathrm{BaF} 3$ cells without any significant effect on the spleen weight. Because $\mathrm{Bcr}-\mathrm{Abl}$ is a chimeric protein, solely and constitutively expressed in BaF3 cells, it is a valuable marker of $\mathrm{BaF} 3$ cell presence. The reduced hepatic expression of $\mathrm{Bcr}-\mathrm{Abl}$ in $\mathrm{BaF} 3-\mathrm{ITF}$ mice compared with $\mathrm{BaF} 3$ mice reflects a lower infiltration of the hepatic parenchyma by the BaF3 cells (Figure 1B) as suggested by histological observation (Supplementary Figure 1A). Hepatic glycogen and triglyceride contents cannot account for the difference in liver weight because they were similar between BaF3 and BaF3-ITF mice (Supplementary Figures $1 \mathrm{~B}$ and $\mathrm{C}$ ). Concordantly to the spleen weight, splenic Bcr-Abl expression was not significantly modified by ITF administration (Figure $1 \mathrm{~B}, P=0.21$ ). The glycolysis pathway becomes predominant in malignant cell lines and tumours and generates lactacte as an end product, whereas lactate dehydrogenase activity in the blood reflects a general cellular turnover and is a negative prognostic factor of leukaemia progression (Montillo et al, 2005; Sonveaux et al, 2008).
Accordingly, plasma L-lactate and plasma lactate dehydrogenase activity were both increased in the presence of the BaF3 tumour, whereas ITF administration decreased these levels (Figure 1C and D). Of note, there were no changes in water or food intake due to the ITF administration (data not shown).

Given that both cancer cell progression and ITF treatment are able to modulate immunity, we measured a panel of cytokines in the plasma. Plasma interleukin 4 (IL-4), interleukin 6 (IL-6), interleukin 10 (IL-10), granulocyte colony-stimulating factor (G-CSF), interleukin 8 (IL-8), monocyte chemo-attractant protein 1 (MCP-1) and RANTES were significantly increased in the BaF3 mice (Figure 1E-K). Inulin-type fructans administration to $\mathrm{BaF} 3$ mice decreased IL-4, IL-8 and MCP-1 levels (Figure 1E, I, J) with no significant effect on IL-10, G-CSF and RANTES levels (Figure 1G, H, K). Interleukin 6 is decreased by ITF treatment, and this is significant by Student's $t$-test $(P=0.04)$. Interferon gamma $($ IFN $\gamma)$ tends to be decreased by ITF feeding $(P=0.05$, Student's $t$-test) (Figure 1L).

\section{Inulin-type fructans administration modifies gut microbiota composition and increases propionate in the portal vein}

We hypothesised that ITF administration reduced $\mathrm{BaF} 3$ cell infiltration and lowered systemic inflammation through gut microbiota modulation. The changes in the gut microbial composition were assessed using DGGE and qPCR of the 16S rRNA gene sequences for total bacteria, Lactobacillus spp., Bifidobacterium spp. (two Gram-positive genera known for antiinflammatory properties (Louis et al, 2007)) and Bacteroides spp. (a predominant Gram-negative genus). The DGGE fingerprints for total bacteria revealed three separate clusters, corresponding to each treatment (Figure 2A). The dendrogram of the DGGE fingerprints showed that the BaF3-ITF mouse fingerprints are closer to those from the control mice compared with the $\mathrm{BaF} 3$ mouse fingerprints. Total bacteria and the Gram-negative Bacteroides were modified by neither the $\mathrm{BaF} 3$ transplantation nor the ITF administration (Figure 2B). Lactobacilli levels were reduced in the BaF3 mice $v s$ the control mice without any impact from the ITF supplementation. Notably, the number of bifidobacteria was below the quantification limit in the mice strain used in this study, that is, $\mathrm{BALB} / \mathrm{c}$ mice.

The caecal content weight tended to decrease in BaF3 mice (not significant in one-way ANOVA, but $P=0.003$ by Student's $t$-test) (Figure $2 \mathrm{C}$ ). We can reasonably suggest that the decreased caecal content weight in BaF3 mice results from the reduced food intake observed during the last 2 days of the experiment (food intake from day 11 to day 13: CT: $9.62 \pm 0.51$; BaF3: $7.02 \pm 0.37 \mathrm{~g}^{*}$; BaF3-ITF: $7.07 \pm 0.31 \mathrm{~g}^{\star} ; n=4,{ }^{\star} P<0.05$ vs CT). However, the caecal content weight was increased in BaF3-ITF mice, even if these mice ate the same amount of food than BaF3 mice. This increase in the caecal content weight reflects the fermentation that occurs upon ITF feeding. The fermentation hypothesis is also supported by the increased caecal tissue weight, only observed in BaF3-ITF mice (Figure 2C). Because we hypothesised that increased SCFA levels could explain the effect of the ITF treatment on cancer progression, we set up a method to quantify the levels of acetate, propionate and butyrate in the portal serum. BaF3 mice tend to exhibit a decreased level of serum propionate and butyrate $(P=0.02$ and 0.05 , respectively, Student's $t$-test). The ITF supplementation did not modify the acetate and butyrate levels, but did induce a two-fold increase of propionate levels (Figure 2D-F).

\section{Short-chain fatty acids decrease BaF3 cell proliferation in vitro}

As typical metabolites of ITF fermentation, SCFA are potentially suitable candidates that could account for the effects of ITF on 
A

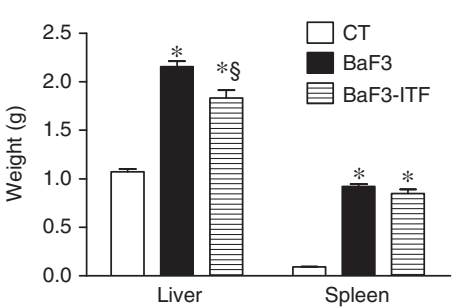

B

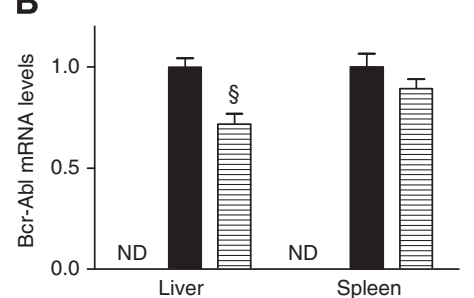

C

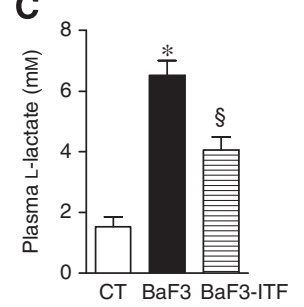

D

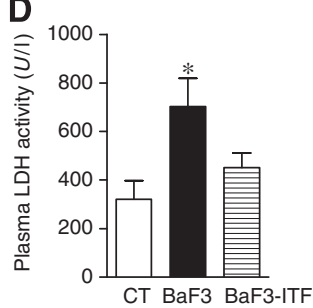

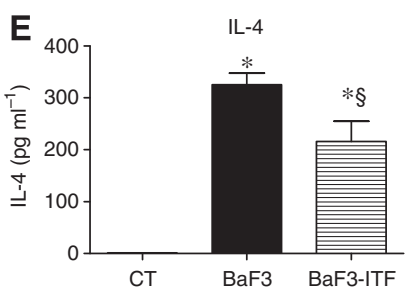
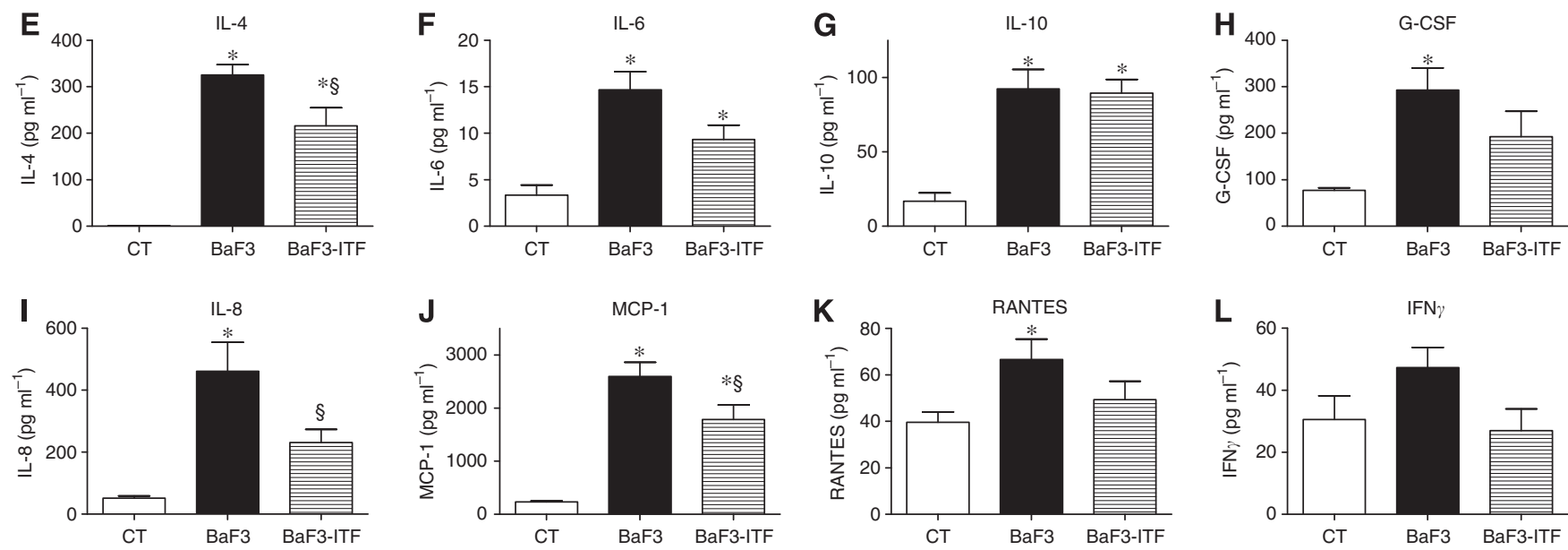

Figure I Inulin-type fructans treatment decreases the hepatic infiltration of the BaF3 cells and systemic inflammation in mice. (A) Liver and spleen weights of the control mice $(\mathrm{CT})$, mice transplanted with BaF3 cells (BaF3) and mice transplanted with BaF3 cells and fed ITF (BaF3-ITF). (B) Bcr-Abl mRNA levels, ND: not detected. (C, D) Plasma L-lactate and lactate dehydrogenase (LDH) activity. (E-L) Plasma levels of IL-4, IL-6, IL- I O, G-CSF, IL-8, MCP- I, RANTES and IFN $\gamma \cdot n=7-8 . * P<0.05$ vs $C T,{ }^{\S} P<0.05$ vs BaF3.

A

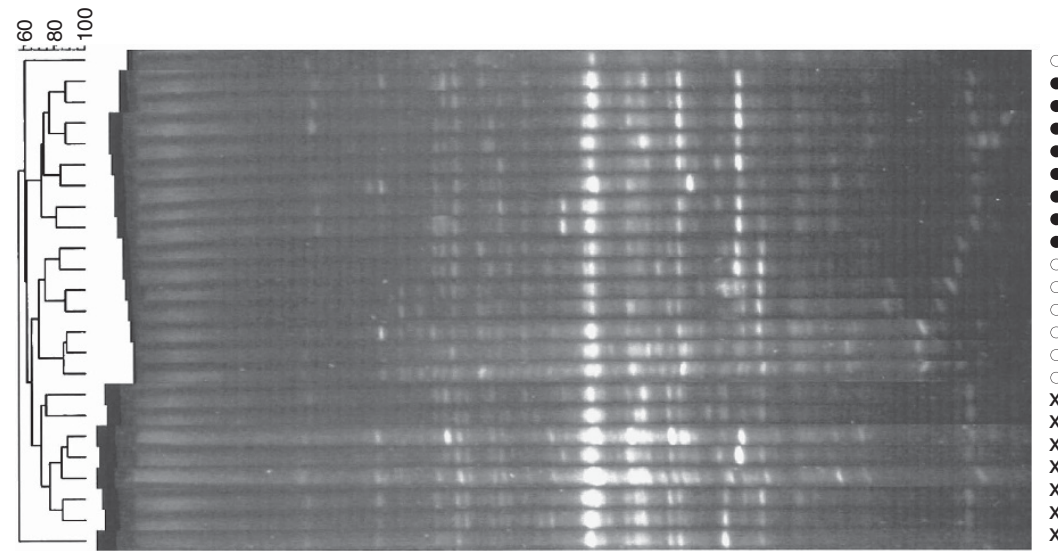

\section{B}

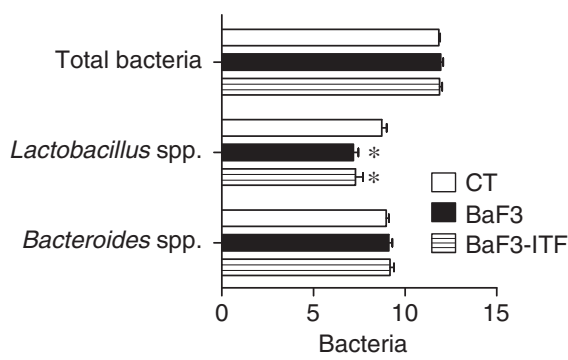

(log [CFU g ${ }^{-1}$ of caecal content])

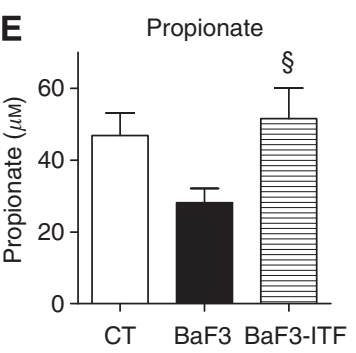

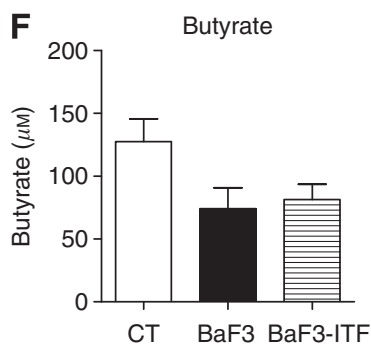

CT BaF3 BaF3-ITF
CT BaF3 BaF3-ITF
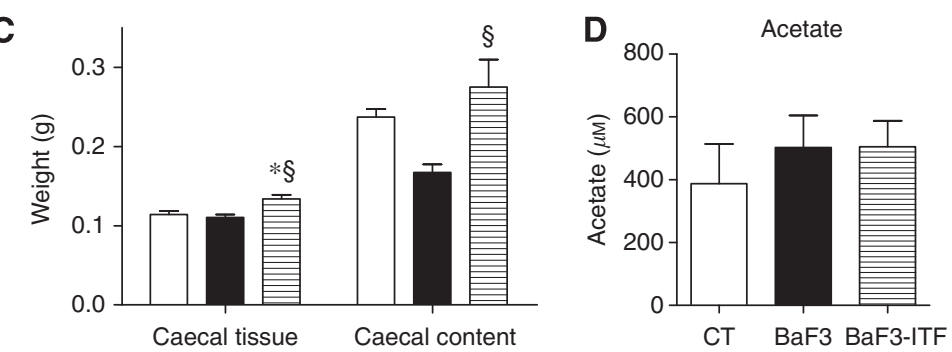

Figure 2 Inulin-type fructans treatment modifies the gut microbiota composition and increases the propionate concentration in the portal vein. (A) Denaturing gradient gel electrophoresis (DGGE) profiles of the bacterial DNA isolated from the caecal content. Cross for the CT mice; closed circle for the BaF3 mice; and open circle for the BaF3-ITF mice. (B) Levels of total bacteria, Lactobacillus spp. and Bacteroides spp. CFU: colony-forming unit. (C) Caecal tissue and caecal content weights. (D-F) Serum acetate, propionate and butyrate concentrations in the portal vein. $n=7-8$. $* P<0.05$ vs $C T$, $\S_{P}<0.05$ vs BaF3. 
$\mathrm{BaF} 3$ cell growth. Indeed, BaF3 cells incubation in the presence of SCFA resulted in a time- and dose-dependent decrease in cell proliferation, as shown by a MTT assay (Figure 3A-C). Notably, SCFA at $10 \mathrm{~mm}$ did not modify the $\mathrm{pH}$ of the medium. Amongst the SCFA, acetate was less potent than propionate or butyrate. $0.2 \mathrm{~mm}$ propionate slightly but significantly reduced $\mathrm{BaF} 3$ cell growth (after $48 \mathrm{~h}$ of incubation, control: $100 \pm 1 \%$; propionate $0,2 \mathrm{~mm}$ : $95 \pm 1 \%$ of control proliferation; $n=3$ in triplicate, $P=0.0032$ ). Given that in vivo ITF increased propionate (but not acetate or butyrate) and reduced $\mathrm{BaF} 3$ cell proliferation, we focused our in vitro studies on the anti-proliferative effect of propionate. Shorter-term incubations, and, therefore, supra-physiological doses, were used to study the potential mechanisms of propionate. BrdU incorporation, reflecting the DNA synthesis, was decreased after 8 and $24 \mathrm{~h}$ of incubation in the presence of 2 and $10 \mathrm{~mm}$ propionate (Figure 3D). These results were confirmed by counting living BaF3 cells incubated in the presence of $2 \mathrm{~mm}$ propionate for 24,48 or $72 \mathrm{~h}$ (Figure 3E).

\section{Propionate reduces $\mathrm{BaF} 3$ cell growth through a cAMP level-dependent pathway}

Propionate is one of the most potent endogenous FFA2 ligands and FFA2 is highly expressed by BaF3 cells (Maslowski et al, 2009). Therefore, we next wanted to determine if a G-protein-coupled receptor pathway (potentially FFA2) was involved in the antiproliferative effect of propionate. FFA2 displays a dual coupling through $\mathrm{Gi}$ and $\mathrm{Gq}$ protein families (Brown et al, 2003; Le Poul et al, 2003). Therefore, BaF3 cells were incubated with U73122, a phospholipase C (PLC) inhibitor. PLC blockade did not blunt the propionate-induced cell growth arrest (Figure 3F; raw data in Supplementary Figure 2). Gi protein inhibits adenylyl cyclase and subsequently reduces cAMP. Therefore, we next investigated if cAMP levels have a role in the anti-proliferative effect of propionate. DibutyrylcAMP, a cAMP analogue, or 3-isobutyl-1methylxanthine (IBMX), an inhibitor of phosphodiesterases, slightly reduced the anti-proliferative action of propionate (Figure 3F; raw data in Supplementary Figure 2). Furthermore, isoproterenol, a $\beta$-adrenoceptor agonist that, among other properties, increases cAMP, also slightly reduced the anti-proliferative effect of propionate (Supplementary Figure 2). Overall, these data suggest that the anti-proliferative effect of propionate is partially dependent on the cAMP level.

\section{Free fatty acid receptor 2 activation decreases mouse and human cell proliferation in vitro}

Finally, we wanted to know whether FFA2 activation is actually able to reduce BaF3 cell growth. To this end, we used CMTB, a potent synthetic FFA2 agonist (Wang et al, 2010), and found that it reduced cell proliferation in a time- and dose-dependent manner (MTT and BrdU assays) (Figure 4A and B). These results were confirmed by microscopic observation after the CMTB treatment (Figure 4C). Furthermore, U73122, dibutyrylcAMP, IBMX and isoproterenol inhibited CMTB-induced cell growth arrest, suggesting that both CAMP- and PLC-dependent pathways are involved in the anti-proliferative effect of CMTB (Supplementary Figure 3).

To support the role of FFA2 in CMTB and propionate effects, we tested their impact on Lewis lung carcinoma (LLC) cells, a cell line that expressed low levels of FFA2 (Supplementary Figure 4A). BaF3 cells were more sensitive to the anti-proliferative action of CMTB and propionate than LLC cells that were almost completely resistant to both treatments, after a 24-h incubation (Supplementary Figure 4B and C). Remarkably, LLC and BaF3 cells were similarly sensitive to doxorubicin, a classical chemotherapeutic agent (Supplementary Figure 4D) (Bray et al, 2010). We can thus reject the hypothesis that LLC cells are generally resistant to any kind of anti-proliferative treatment.

To further extend the concept of FFA2 as a new cancer therapeutic target, we studied the effect of CMTB on two human cancer cell lines, U937 cells and K562 cells. CMTB reduced the growth of both cell lines. The U937 cells, which express a 25 -fold
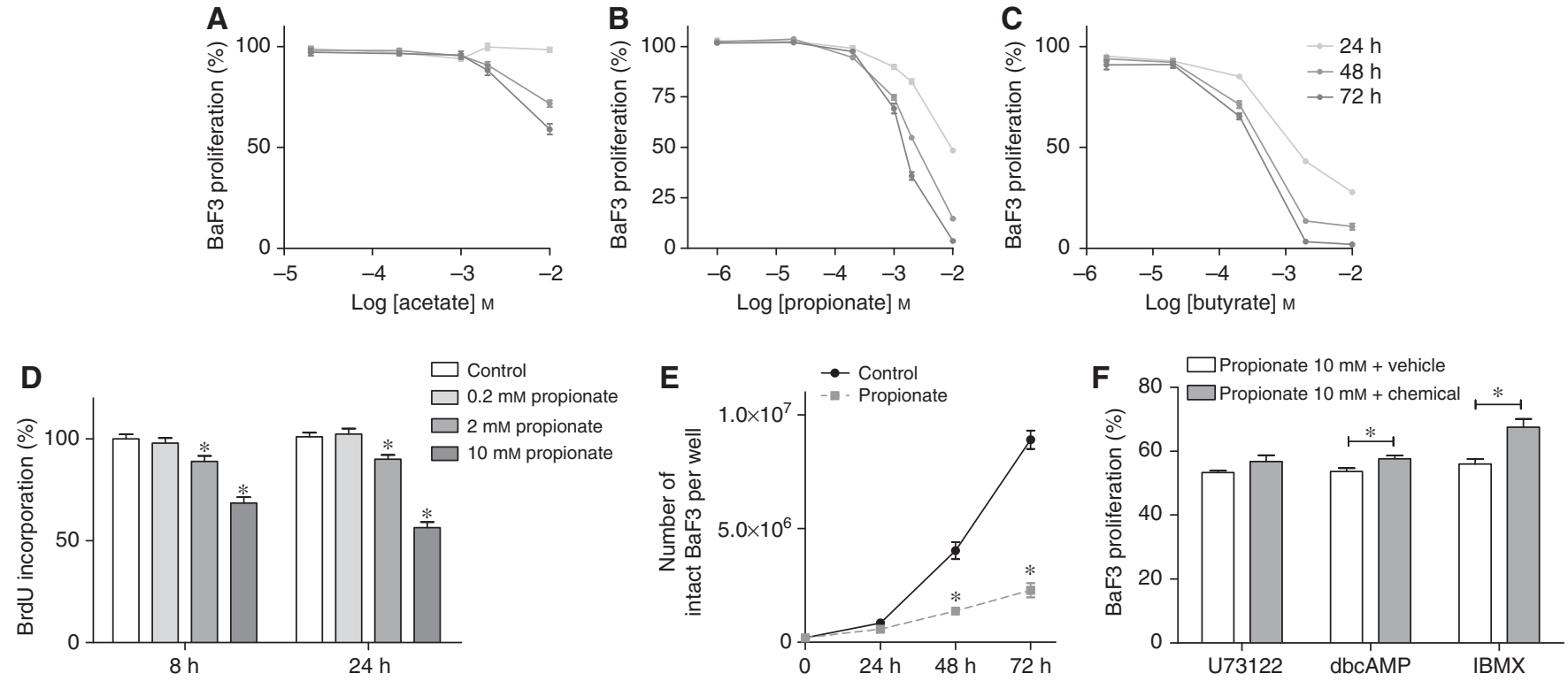

Figure 3 Short-chain fatty acids decrease BaF3 cell proliferation in vitro. (A-C) BaF3 cells were incubated in the absence or presence of acetate, propionate, butyrate for 24, 48 and $72 \mathrm{~h}$ before performing the MTT assay. (D) BrdU incorporation after 8 or $24 \mathrm{~h}$ incubation in the absence or presence of propionate. (E) Manual counting of BaF3 cells after 24, 48 and $72 \mathrm{~h}$ in the absence or presence of 2 mm propionate. (F) BaF3 cells were pre-incubated in the absence or presence of the indicated drug (U73|22, $2 \mu \mathrm{M}$, I h; dbcAMP I mM, $30 \mathrm{~min}$; IBMX, 316 $6 \mathrm{M}$, I h) and then incubated in the absence or presence of propionate for $24 \mathrm{~h}$. Data are expressed as a percentage of the control performed for each pharmacological condition in the absence of propionate. MTT assay. The graphs represent data obtained from at least three independent experiments performed in triplicate. $* P<0.05$ vs the control. For F, each set of data was analysed by two-way ANOVA with Bonferroni post hoc test taking into account control values. 


\section{A}

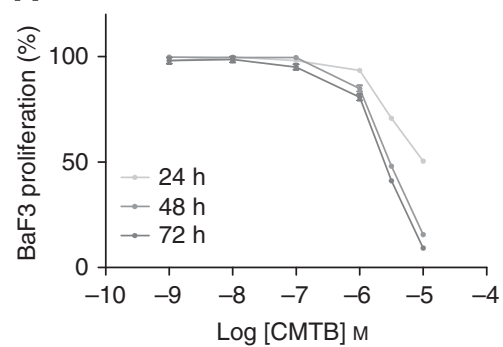

B

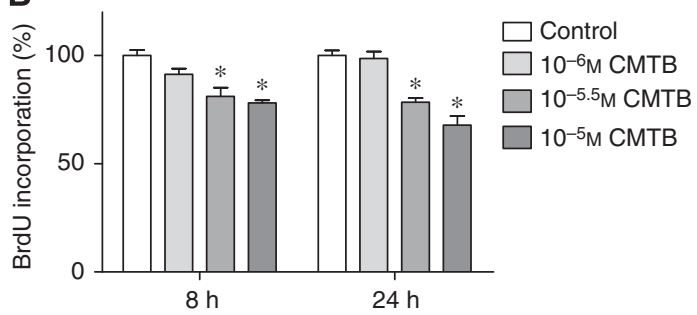

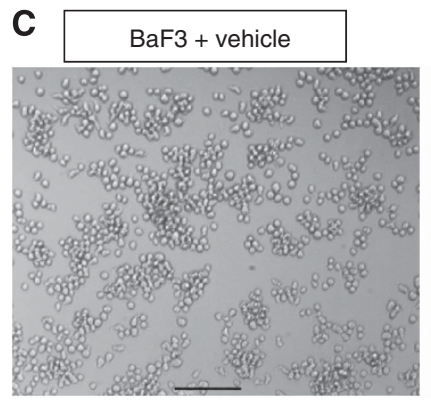
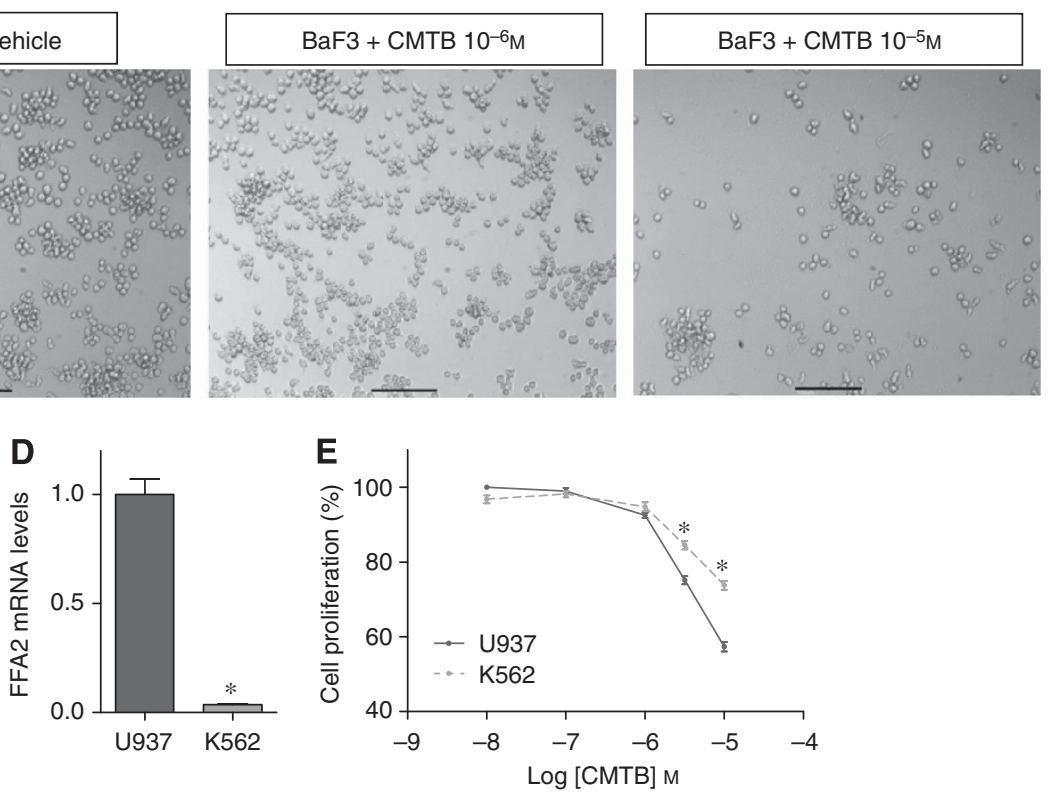

Figure 4 Free fatty acid receptor 2 (FFA2) activation decreases the proliferation of BaF3 cells in vitro. (A) BaF3 cells were incubated in the absence or presence of CMTB for 24, 48 and $72 \mathrm{~h}$ before performing MTT assay. (B) BrdU incorporation after 8 or $24 \mathrm{~h}$ incubation in the absence or presence of CMTB. $* P<0.05$ vs the control. (C) Microscopic pictures of BaF3 cells incubated in the absence or presence of CMTB. Scale bar $=100 \mu \mathrm{M}$. (D) Free fatty acid receptor 2 (FFA2) mRNA levels in U937 and K562 cells. *P<0.05 vs U937. $n=1$ in triplicate. (E) U937 and K562 cells were incubated in the absence or presence of CMTB for $24 \mathrm{~h}$. MTT assay. ${ }^{*} P<0.05$ vs U937. The MTT and BrdU assays graphs represent data obtained from at least three independent experiments performed in triplicate.

higher level of the FFA2 transcript as compared with the K562 cells, are more sensitive to CMTB treatment (Figure 4D and E).

\section{DISCUSSION}

In this study, we demonstrate in an acute leukaemia mouse model that dietary ITF administration reduces cancer cell infiltration into the liver and reduces the inflammation associated with cancer progression. In vitro, we demonstrated that the anti-proliferative effect of propionate was partially cAMP level-dependent, and that FFA2 activation altered cancer cell proliferation. These results support the idea of a role for gut microbiota in the control of systemic cancer.

The anti-inflammatory effect of dietary ITF was demonstrated in pathological conditions, such as obesity, diabetes or intestinal inflammation (Cherbut et al, 2003; Cani et al, 2006; Cani et al, 2007) and this effect was related to the microbial changes induced by ITF feeding, namely to an increase in bifidobacteria and lactobacilli (Louis et al, 2007). The ITF fermentation, reflected by the increased caecal content and tissue weights in BaF3-ITF mice, is accompanied by changes in the total gut microbiota composition, without changing lactobacilli and bifidobacteria levels. Therefore, at this stage, we cannot attribute the anti-inflammatory effect of ITF to the changes in the level of lactic acid bacteria, as suggested in other models.
The gut microbiota could influence BaF3 cell progression by changing its metabolome. We hypothesised that a gut microbiotaderived metabolite, that is increased upon ITF treatment and specifically targets liver tissue, could mediate a protective effect. Propionate fulfills all of these requirements because it is increased in the portal vein of ITF-fed rats (Daubioul et al, 2002) and is mostly taken up by the liver (Guarner and Malagelada, 2003). In the present in vivo study, propionate levels were increased in the portal blood of the BaF3-ITF mice. Of note, propionate and butyrate levels tended to be decreased upon cancer. This could be explained by the altered gut microbiota composition or by the decreased food intake observed at the end of the treatment, which could have lessened the substrate supply.

Corroborating the in vivo study, in vitro studies showed an antiproliferative effect of propionate on BaF3 cells. Therefore, we suggest that propionate is, amongst the SCFA, the most likely mediator of the ITF anti-tumour effect. We can speculate that propionate uptake by the liver explains why ITF has an impact on $\mathrm{BaF} 3$ cell progression in the liver and not in the spleen.

In human colon cancer cells and neutrophils, the antiproliferative capacity of SCFA has been associated with the SCFA ability to inhibit histone deacetylase activity (Hinnebusch et al, 2002; Aoyama et al, 2010). Butyrate was the most powerful inhibitor, whereas propionate demonstrated an intermediate profile. Acetate did not influence the histone deacetylase activity (Hinnebusch et al, 2002; Aoyama et al, 2010). In our in vitro 
experiments, both acetate and propionate reduce BaF3 cell proliferation. This led us to hypothesise that targets other than histone deacetylases might be involved in the in vitro antiproliferative effect of SCFA, namely FFA2 and FFA3. Quantitative real-time PCR revealed that FFA3 expression in the BaF3 cells was approximately 100 -fold lower than FFA2 expression (data not shown). Therefore, we focused on the potential implication of FFA2. In view of the comparison of cell lines with different level of expression of FFA2 (LLC and BaF3 cells), we postulate that FFA2 might be involved in the anti-proliferative action of propionate. Free fatty acid receptor 2 can couple with the Gi and Gq pathways (Brown et al, 2003; Le Poul et al, 2003). Blocking the Gq-PLC pathway through a PLC inhibitor did not counteract the antiproliferative effect of propionate. Mimicking an increase in cAMP level (by dibutyrylcAMP) and indirectly modifying cAMP level (by IBMX or isoproterenol) significantly, but only slightly, reduces the anti-proliferative effect of propionate. Therefore, we propose that the anti-proliferative activity of propionate could be PLCindependent and partially cAMP level-dependent. Phenylacetamide derivatives, such as CMTB, have been recently described as FFA2 agonists and are inactive against a panel of GPCRs (included FFA3) at concentrations of up to $30 \mu \mathrm{m}$ (Lee et al, 2008; Wang et al, 2010). Here, we establish that FFA2 activation by CMTB reduces BaF3 cell growth through PLC- and cAMP level-dependent pathways. Importantly, evidence from pharmacological studies suggests that the signalling pathways downstream of FFA2 are differentially activated by synthetic and endogenous ligands (Lee et al, 2008). In fact, molecular modelling analysis revealed that CMTB and endogenous ligands do not bind to the same site, and this could be related to the fact that CMTB and propionate differentially activate the $\mathrm{Gi}$ and $\mathrm{Gq}$ pathways. Indeed, CMTB analogues equally activate $\mathrm{Gq}$ and $\mathrm{Gi}$ pathways, whereas propionate is much more potent on the Gi than the Gq pathway (Lee et al, 2008). Additional work is needed to evaluate the molecular

\section{REFERENCES}

Alric R, Cociglio M, Blayac JP, Puech R (1981) Performance evaluation of a reversed-phase, high-performance liquid chromatographic assay of valproic acid involving a "solvent demixing" extraction procedure and precolumn derivatisation. J Chromatogr 224: 289-299

Aoyama M, Kotani J, Usami M (2010) Butyrate and propionate induced activated or non-activated neutrophil apoptosis via HDAC inhibitor activity but without activating GPR-41/GPR-43 pathways. Nutrition 26(6): 653-661

Bindels LB, Beck R, Schakman O, Martin JC, De Backer FC, Sohet FM, Dewulf EM, Pachikian BD, Neyrinck AM, Thissen JP, Verrax J, Calderon PB, Pot B, Grangette C, Cani PD, Delzenne NM (2012) Restoring specific lactobacilli levels decreases inflammation and muscle atrophy markers in an acute leukemia mouse model. PLoS One 7(6): e37971

Bray J, Sludden J, Griffin MJ, Cole M, Verrill M, Jamieson D, Boddy AV (2010) Influence of pharmacogenetics on response and toxicity in breast cancer patients treated with doxorubicin and cyclophosphamide. $\mathrm{Br} J$ Cancer 102(6): 1003-1009

Brown AJ, Goldsworthy SM, Barnes AA, Eilert MM, Tcheang L, Daniels D, Muir AI, Wigglesworth MJ, Kinghorn I, Fraser NJ, Pike NB, Strum JC, Steplewski KM, Murdock PR, Holder JC, Marshall FH, Szekeres PG, Wilson S, Ignar DM, Foord SM, Wise A, Dowell SJ (2003) The Orphan G protein-coupled receptors GPR 41 and GPR43 are activated by propionate and other short chain carboxylic acids. J Biol Chem 278(13): 11312-11319

Busserolles J, Gueux E, Rock E, Demigne C, Mazur A, Rayssiguier Y (2003) Oligofructose protects against the hypertriglyceridemic and pro-oxidative effects of a high fructose diet in rats. J Nutr 133(6): 1903-1908

Cani PD, Knauf C, Iglesias MA, Drucker DJ, Delzenne NM, Burcelin R (2006) Improvement of glucose tolerance and hepatic insulin sensitivity by oligofructose requires a functional glucagon-like Peptide 1 receptor. Diabetes 55(5): 1484-1490

Cani PD, Neyrinck AM, Fava F, Knauf C, Burcelin RG, Tuohy KM, Gibson GR, Delzenne NM (2007) Selective increases of bifidobacteria in gut microflora improve high-fat-diet-induced diabetes in mice through a characteristics of FFA2 at the protein level in FFA2-expressing cancer cells, before really evaluating the contribution of the binding of propionate to FFA2 in its anti-proliferative activity.

Finally, using human leukaemic cell lines, we have extended the concept of FFA2 activation as a tumour-suppressive therapy. Tang et al (2011b) recently reported that increasing the FFA2 expression in human colon cancer cells by plasmid transfection sensitises them to the action of propionate. Our results suggest that FFA2 is of therapeutic interest for the treatment of different hematopoietic cancers.

In conclusion, both in vivo and in vitro approaches support the therapeutic potential of nutrients targeting the gut microbiota in the control of leukaemic disease. We propose that propionate production could be one of the gut microbial functions responsible for the anti-tumour effect of prebiotic nutrients. Furthermore, our data support the therapeutic interest in the pharmacological activation of FFA2 to control cancer cell proliferation.

\section{ACKNOWLEDGEMENTS}

We thank FM Sohet, BP Pachikian and F De Backer for helpful discussion and technical support. LBB is a Research Fellow, JV is a Scientific Research Worker and PS and PDC are Research Associates from the FRS-FNRS (Fond National de la Recherche Scientifique) in Belgium. The RINH, University of Aberdeen, receives support from the Scottish Government (RESAS). GGM is grateful to the Universite catholique de Louvain and to the FRSFNRS (Fond de la Recherche Scientifique) for a FSR and a FRFC (2.4555.08) grant, respectively. PDC and NMD are recipients of FRS-FNRS grant (1.5105.11 and 1.A420.09).

Supplementary Information accompanies the paper on British Journal of Cancer website (http://www.nature.com/bjc)

mechanism associated with endotoxaemia. Diabetologia 50(11): 2374-2383

Cherbut C, Michel C, Lecannu G (2003) The prebiotic characteristics of fructooligosaccharides are necessary for reduction of TNBS-induced colitis in rats. J Nutr 133(1): 21-27

Cox MA, Jackson J, Stanton M, Rojas-Triana A, Bober L, Laverty M, Yang X, Zhu F, Liu J, Wang S, Monsma F, Vassileva G, Maguire M, Gustafson E, Bayne M, Chou CC, Lundell D, Jenh CH (2009) Short-chain fatty acids act as antiinflammatory mediators by regulating prostaglandin $\mathrm{E}(2)$ and cytokines. World J Gastroenterol 15(44): 5549-5557

Daubioul C, Rousseau N, Demeure R, Gallez B, Taper H, Declerck B, Delzenne N (2002) Dietary fructans, but not cellulose, decrease triglyceride accumulation in the liver of obese Zucker fa/fa rats. $J$ Nutr 132(5): 967-973

Everard A, Lazarevic V, Derrien M, Girard M, Muccioli GM, Neyrinck AM, Possemiers S, Van HA, Francois P, de Vos WM, Delzenne NM, Schrenzel J, Cani PD (2011) Responses of gut microbiota and glucose and lipid metabolism to prebiotics in genetic obese and diet-induced leptinresistant mice. Diabetes 60(11): 2775-2786

Fiskus W, Pranpat M, Bali P, Balasis M, Kumaraswamy S, Boyapalle S, Rocha K, Wu J, Giles F, Manley PW, Atadja P, Bhalla K (2006) Combined effects of novel tyrosine kinase inhibitor AMN107 and histone deacetylase inhibitor LBH589 against Bcr-Abl-expressing human leukemia cells. Blood 108(2): 645-652

Guarner F, Malagelada JR (2003) Gut flora in health and disease. Lancet 361(9356): $512-519$

Hinnebusch BF, Meng S, Wu JT, Archer SY, Hodin RA (2002) The effects of short-chain fatty acids on human colon cancer cell phenotype are associated with histone hyperacetylation. J Nutr 132(5): 1012-1017

Hong YH, Nishimura Y, Hishikawa D, Tsuzuki H, Miyahara H, Gotoh C, Choi KC, Feng DD, Chen C, Lee HG, Katoh K, Roh SG, Sasaki S (2005) Acetate and propionate short chain fatty acids stimulate adipogenesis via GPCR43. Endocrinology 146(12): 5092-5099 
Kondegowda NG, Meaney MP, Baker C, Ju YH (2011) Effects of nondigestible carbohydrates on the growth of estrogen-dependent human breast cancer (MCF-7) tumors implanted in ovariectomized athymic mice. Nutr Cancer 63(1): 55-64

Le Poul E, Loison C, Struyf S, Springael JY, Lannoy V, Decobecq ME, Brezillon S, Dupriez V, Vassart G, Van DJ, Parmentier M, Detheux M (2003) Functional characterization of human receptors for short chain fatty acids and their role in polymorphonuclear cell activation. $J$ Biol Chem 278(28): 25481-25489

Lee T, Schwandner R, Swaminath G, Weiszmann J, Cardozo M, Greenberg J, Jaeckel P, Ge H, Wang Y, Jiao X, Liu J, Kayser F, Tian H, Li Y (2008) Identification and functional characterization of allosteric agonists for the G protein-coupled receptor FFA2. Mol Pharmacol 74(6): 1599-1609

Louis P, Scott KP, Duncan SH, Flint HJ (2007) Understanding the effects of diet on bacterial metabolism in the large intestine. J Appl Microbiol 102(5): 1197-1208

Maslowski KM, Vieira AT, Ng A, Kranich J, Sierro F, Yu D, Schilter HC, Rolph MS, Mackay F, Artis D, Xavier RJ, Teixeira MM, Mackay CR (2009) Regulation of inflammatory responses by gut microbiota and chemoattractant receptor GPR43. Nature 461(7268): 1282-1286

Montillo M, Hamblin T, Hallek M, Montserrat E, Morra E (2005) Chronic lymphocytic leukemia: novel prognostic factors and their relevance for risk-adapted therapeutic strategies. Haematologica 90(3): 391-399

Neish AS (2009) Microbes in gastrointestinal health and disease. Gastroenterology 136(1): 65-80

Pool-Zobel BL, Sauer J (2007) Overview of experimental data on reduction of colorectal cancer risk by inulin-type fructans. J Nutr 137(11 Suppl): 2580S-2584S

Ren R (2005) Mechanisms of BCR-ABL in the pathogenesis of chronic myelogenous leukaemia. Nat Rev Cancer 5(3): 172-183

Siavoshian S, Segain JP, Kornprobst M, Bonnet C, Cherbut C, Galmiche JP, Blottiere HM (2000) Butyrate and trichostatin A effects on the proliferation/differentiation of human intestinal epithelial cells: induction of cyclin D3 and p21 expression. Gut 46(4): 507-514
Sonveaux P, Vegran F, Schroeder T, Wergin MC, Verrax J, Rabbani ZN, De Saedeleer CJ, Kennedy KM, Diepart C, Jordan BF, Kelley MJ, Gallez B, Wahl ML, Feron O, Dewhirst MW (2008) Targeting lactate-fueled respiration selectively kills hypoxic tumor cells in mice. J Clin Invest 118(12): 3930-3942

Sundstrom C, Nilsson K (1976) Establishment and characterization of a human histiocytic lymphoma cell line (U-937). Int J Cancer 17(5): 565-577

Tang Y, Chen Y, Jiang H, Nie D (2011a) Short-chain fatty acids induced autophagy serves as an adaptive strategy for retarding mitochondriamediated apoptotic cell death. Cell Death Differ 18(4): 602-618

Tang Y, Chen Y, Jiang H, Robbins GT, Nie D (2011b) G-protein-coupled receptor for short-chain fatty acids suppresses colon cancer. Int J Cancer 128(4): 847-856

Taper HS, Delzenne NM, Roberfroid MB (1997) Growth inhibition of transplantable mouse tumors by non-digestible carbohydrates. Int J Cancer 71(6): 1109-1112

Tolhurst G, Heffron H, Lam YS, Parker HE, Habib AM, Diakogiannaki E, Cameron J, Grosse J, Reimann F, Gribble FM (2012) Short-chain fatty acids stimulate glucagon-like peptide-1 secretion via the G-proteincoupled receptor FFAR2. Diabetes 61(2): 364-371

Vinolo MA, Ferguson GJ, Kulkarni S, Damoulakis G, Anderson K, Bohlooly Y, Stephens L, Hawkins PT, Curi R (2011) SCFAs induce mouse neutrophil chemotaxis through the GPR43 receptor. PLoS One 6(6): e21205

Wang Y, Jiao X, Kayser F, Liu J, Wang Z, Wanska M, Greenberg J, Weiszmann J, Ge H, Tian H, Wong S, Schwandner R, Lee T, Li Y (2010) The first synthetic agonists of FFA2: discovery and SAR of phenylacetamides as allosteric modulators. Bioorg Med Chem Lett 20(2): 493-498

(c) (1) (2) This work is licensed under the Creative Commons

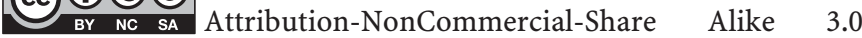
Unported License. To view a copy of this license, visit http:// creativecommons.org/licenses/by-nc-sa/3.0/ 